\title{
Spectroscopic study of the open cluster Blanco $1^{\star}$
}

\author{
J. F. González and H. Levato
}

\author{
Instituto de Ciencias Astronómicas, de la Tierra y del Espacio, Casilla 467, 5400 San Juan, Argentina \\ e-mail: fgonzalez@casleo.gov.ar
}

Received 26 June 2009 / Accepted 17 August 2009

\begin{abstract}
Aims. As a part of our program on binaries in open clusters, we present a spectroscopic study of the bright stars of Blanco 1 aimed at detecting and characterizing spectroscopic binaries.

Methods. Forty five stars previously mentioned as cluster candidates, plus another 24 stars in a wider region around the cluster were observed repeatedly during 6 years, with a spectral resolving power 13300 . Radial velocities were measured by cross-correlations. Results. We obtained a mean cluster velocity of $6.2 \pm 0.3 \mathrm{~km} \mathrm{~s}^{-1}$ and determined kinematic membership. Eleven spectroscopic binaries were detected, and orbital solutions are presented for eight of them. Six binaries are confirmed to be members of the cluster. All of them are single-lined spectroscopic systems with periods in the range 1.9-1380 days. Considering all suspected binaries, the cluster binary frequency amounts to about $50 \%$.
\end{abstract}

Key words. open clusters and associations: individual: Blanco 1 - binaries: spectroscopic - techniques: radial velocities

\section{Introduction}

While counting stars of spectral type A0 in Kapteyn's selected areas, Blanco (1949) found an area with star density about five times the average value at that galactic latitude. A plot of spectral classes against apparent magnitude allowed him to identify a sequence of stars in the HR diagram. Proper motions confirmed that those stars were part of an open cluster. He had discovered a galactic open cluster that is very unusual in its high galactic latitude.

The first extensive photometric study in the region was carried out by Abraham de Epstein \& Epstein (1985). They found that around 150 stars out of the 1500 stars observed belong to the unevolved main sequence of the cluster. They determined a mean modulus of $6.9 \mathrm{mag}$ and a small reddening. They reported also two gaps in the main sequence around $B-V=0.06-0.30$ and $B-V=1.0 \mathrm{mag}$.

Westerlund et al. (1988) observed 130 stars photoelectrically, identifying 32 stars as members of the cluster, in addition to a number of $\mathrm{F}$ and $\mathrm{G}$ type stars considered to be probable members. They found an interstellar reddening $E(B-V)=0.02 \mathrm{mag}$ and a distance modulus of $V_{o}-M_{v}=6.93 \pm 0.06 \mathrm{mag}$.

Modern work on Blanco 1 started with the paper by Panagi et al. (1994), who made a spectroscopic study of 115 stars that are probably members of the cluster. From the intensity of the Li $(6708 \AA)$ line, they concluded that the age of the cluster is close to that of $\alpha$ Persei, and they redefined the cluster distance as 246 pc. However, Panagi \& O'Dell (1997) extended this work and found, from the number of $\mathrm{H} \alpha$-emision line stars among $\mathrm{K}$ dwarfs, that Blanco 1 is slightly older than the Pleiades.

Edvardson et al. (1995) determined chemical abundances and radial velocities. They found an overall metallicity of

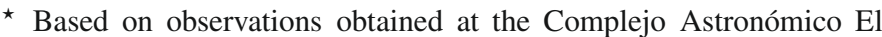
Leoncito (CASLEO), Argentina.
}

$[\mathrm{Fe} / \mathrm{H}]=+0.24$ for the cluster, with an unusual chemical abundance pattern. More recently, Ford et al. (2005) found a metallicity close to solar: $[\mathrm{Fe} / \mathrm{H}]=+0.04 \pm 0.04$. For its age, the cluster is unusually far from the galactic disk $(240 \mathrm{pc})$, and its radial velocity $\left(+5.5 \mathrm{~km} \mathrm{~s}^{-1}\right.$, Mermilliod et al. 2008) would indicate that it is close to its maximum distance from the galactic plane. Edvardson et al. (1995) suggest that the cluster was formed in the galactic disk $45 \mathrm{Myr}$ ago by the interaction of a high-velocity cloud with the interstellar medium and that its formation may be connected with that of Gould's Belt. Blanco 1 became still more interesting because of the discovery of X ray emission by Randich (1997). Using HRI ROSAT, Micela et al. (1997) found a high number of X-ray sources consistent with the young age of the cluster, some of which showed variability on time scales from hours to one year.

Subsequent X-ray studies were published by Micela et al. (1999) and Pillitteri et al. (2003, 2004, 2005), who determined the X-ray luminosity function of the cluster and detected flares and X-ray variability using XMM-Newton. Cargile et al. (2009) published a new analysis of the ROSAT and XMM-Newton X-ray surveys of Blanco 1, utilizing new BVIc photometric and proper motion data sets. They matched optical counterparts to 47 X-ray sources associated with Blanco 1 cluster members, six of which were listed in previous X-ray studies as cluster nonmembers. This new catalog of optical counterparts to $\mathrm{X}$-ray sources clearly traces the Blanco 1 main sequence in a color-magnitude diagram, extending from early $\mathrm{G}$ to mid-M spectral types. Moraux et al. (2007) studied the lower mass function from 30 Jupiter masses up to 3 solar masses. Mermilliod et al. (2008) published a binarity and kinematic membership study carried out with the CORAVEL spectrograph.

In this paper we present the results of a long-term spectroscopic study carried out at CASLEO as a part of a wider project aimed to derive the frequency and properties of binaries 
Table 1. Radial velocity measurements (fragment).

\begin{tabular}{crrr}
\hline \hline Star & HJD-2450000 & $\begin{array}{r}\mathrm{RV} \\
\mathrm{km} \mathrm{s}^{-1}\end{array}$ & $\begin{array}{r}\text { err } \\
\mathrm{km} \mathrm{s}^{-1}\end{array}$ \\
\hline W3 & 2842.84581 & -11.06 & 0.73 \\
W3 & 2859.86137 & -10.79 & 0.75 \\
W3 & 2862.92307 & -10.64 & 0.76 \\
W3 & 2865.91862 & -10.62 & 0.80 \\
W4 & 2842.82617 & 7.55 & 0.84 \\
W4 & 3217.77759 & 8.97 & 0.75 \\
W8 & 2474.86347 & 4.39 & 0.84 \\
W8 & 3217.75920 & 5.88 & 0.77 \\
W8 & 3632.80986 & 3.84 & 1.14 \\
\hline
\end{tabular}

in open clusters. Preliminary results on Blanco 1 were reported by Levato et al. (2004) and González \& Levato (2006a). In Sect. 2 we describe the observations and in Sect. 3 present the analysis of spectroscopic binaries. Section 4 deals with the cluster kinematics, while the main results are summarized in Sect. 5.

\section{Observations and radial velocity measurements}

Multi-epoch spectroscopic observations were collected for selected stars in the field of Blanco 1 from June 2002 to September 2008. Our primary sample contains all the stars brighter than $V=10.5$, within a radius $1.5 \mathrm{deg}$ of the cluster, whose location in the color-magnitude diagram is compatible with the cluster main sequence. This sample of 45 stars included also a few fainter $(V=10.5-11.3 \mathrm{mag})$ stars. In addition, we obtained one or two spectra for another 24 bright stars in a wider region up to $4 \mathrm{deg}$ from the cluster center. We used the echelle spectrograph REOSC mounted at the Cassegrain focus of the $2.1 \mathrm{~m}$ telescope at CASLEO. The spectra cover the spectral range 3600-6000 $\AA$ with a resolving power of 13300 , corresponding to 2.5 pixels. Data reduction was carried out using standard IRAF tasks.

Radial velocities (RVs) were measured by cross-correlations using synthetic spectra from the grid of Munari et al. (2005) as templates. These spectra cover the whole spectral range of our observations with a resolution of 20000 . For the crosscorrelation of rapidly rotating program stars, a convolution with an appropriate rotational profile was applied to the template. Individual radial velocity measurements for all the observed stars are in Table 1, available in its entirety in electronic form. The identifiers used here correspond to the numbering of Westerlund et al. (1988) (prefix W), Epstein (1968) (prefix E), and Abraham de Epstein \& Epstein (1985) (prefix SZ). Some bright stars not included in previous works of the cluster are identified with HD or Tycho (ESA 1997) numbers.

RV errors were calculated as the quadratic sum of two contributions. On the one hand, we considered the measurement error provided by the IRAF task fxcor, which depends mainly on the spectrum noise and the possible mismatch between object and template spectra. On the other hand, we included a contribution that represents the probable shift due to the centering error of the star in the slit or wavelength calibration errors. This contribution was evaluated for each observing run by calculating the rms of measurements of standard stars or high-quality constant-velocity stars. The typical value for this error was $0.6-1.5 \mathrm{~km} \mathrm{~s}^{-1}$.

We obtained 3-5 spectra for every star in our sample of cluster stars and calculated the statistical quantity $\chi^{2}$, from which we determined the probability $P\left(\chi^{2}, n\right)$ that the star is constant. Stars for which $P\left(\chi^{2}, n\right)<0.005$ are classified as RV variables and stars with $P\left(\chi^{2}, n\right)>0.05$ as constant, while stars with values in the range $0.005-0.05$ are considered suspected of variability.

Mean RVs, variability, and membership information are listed in Table 2. Columns 1 to 3 list identification numbers and visual magnitude. Columns 4 to 6 give the mean RV, the error of the mean RV, and the number of measurements. Column 7 is the probability of being RV constant and Col. 8 the variability assignment: constant (c), suspected variable (?), variable (v). Column 9 lists the spectral type as determined by comparison with observed spectra of standard stars, and Col. 10 the rotational velocity. Columns 11 to 13 give membership information: according to RVs, to proper motions, and the adopted membership.

Two stars presented composite spectra, one of them with very weak secondary lines (HD 224113) and the other with both components strongly blended (W65). In both cases, it was not possible to measure the two components with standard cross-correlations; instead, we applied the method described in González \& Levato (2006b).

Rotational velocities were derived by measuring the width of the peak in the cross-correlation function of the object against a low-rotation template. The calibration of the FWHM of the correlation peak versus $v \sin i$ was obtained by measuring lowrotation spectra previously convolved with different rotational profiles. Stars for which $v \sin i$ is not consigned in Table 2 have rotational velocity below our spectral resolution $\left(<25 \mathrm{~km} \mathrm{~s}^{-1}\right)$.

\section{Spectroscopic binaries}

After the first observing runs, we identified suspected variable stars and afterwards we monitored their radial velocities to obtain their orbits. Eleven stars from our cluster star sample were confirmed to be variable: W37, W42, W47, W57, W61, W65, W71, W76, W86, W96, and E57. Furthermore, stars W75, $\mathrm{W} 121$, and W126 are classified as probably variable. All of them are single-lined stars, with the only exception of W65, which is suspected of being an SB2 as discussed below. That secondary components are not visible in our spectra for the remaining binaries would indicate that the luminosity ratios are very probably less than 0.1 for all of them.

We have identified orbital periods and fitted Keplerian orbits for the seven single-lined binaries listed in Table 3. Their RV curves are shown in Fig. 1. Table 3 and Fig. 1 also include the eclipsing binary AL Scl (HD 224113), which is located at $2.7 \mathrm{deg}$ from the cluster and whose connection with the cluster is discussed in a separate paragraph below.

Some additional stars classified as RV constant according to our measurements should be considered probable variable stars considering the RV difference between our observations and those of previous works. Stars W88, W124, and E54 present significant RV difference with respect to the results of Bond \& Perry (1971). The comparison of our mean RVs with those obtained by Mermilliod et al. (2008) for ten stars in common classified as RV constant stars reveals the variability of star W54 $\left(\Delta \mathrm{RV}=6.2 \pm 1.8 \mathrm{~km} \mathrm{~s}^{-1}\right)$

In the following we describe some particular objects.

W37: it is clearly an RV variable, although the present observations do not allow identifying the period unambiguously. The cross-correlation function does not show asymmetries. It is classified as a photometric double star by Mermilliod et al. (2008) according to its position in the color-magnitude diagram (see Fig. 2). 
Table 2. Mean radial velocities, variability, and membership.

\begin{tabular}{|c|c|c|c|c|c|c|c|c|c|c|c|c|c|}
\hline \multirow{2}{*}{$\begin{array}{l}\text { Star } \\
\text { (1) }\end{array}$} & \multirow{2}{*}{$\begin{array}{l}\text { HD } \\
\text { (2) }\end{array}$} & \multirow{2}{*}{$\begin{array}{c}V \\
\text { mag } \\
(3)\end{array}$} & \multirow{2}{*}{$\begin{array}{c}\langle\mathrm{RV}\rangle \\
\mathrm{km} \mathrm{s}^{-1} \\
(4)\end{array}$} & \multirow{2}{*}{$\begin{array}{c}\text { err } \\
\mathrm{km} \mathrm{s}^{-1} \\
(5)\end{array}$} & \multirow{2}{*}{$\begin{array}{l}N \\
\text { (6) }\end{array}$} & \multirow{2}{*}{$\begin{array}{c}P\left(\chi^{2}\right) \\
\% \\
(7)\end{array}$} & \multirow{2}{*}{$\begin{array}{c}\text { var } \\
\text { const } \\
(8)\end{array}$} & \multirow{2}{*}{$\begin{array}{l}\text { Sp. type } \\
\text { (9) }\end{array}$} & \multirow{2}{*}{$\begin{array}{c}v \sin i \\
\mathrm{~km} \mathrm{~s}^{-1} \\
(10)\end{array}$} & \multicolumn{3}{|c|}{ Membership } & \multirow{2}{*}{$\begin{array}{c}\text { Notes } \\
(14) \\
\end{array}$} \\
\hline & & & & & & & & & & $\begin{array}{c}\text { RV } \\
\text { (11) }\end{array}$ & $\begin{array}{l}\text { PM } \\
\text { (12) }\end{array}$ & Adop. & \\
\hline W3 & 224515 & 8.4 & -10.8 & 0.4 & 4 & 97 & $\mathrm{c}$ & K0III & $\ldots$ & $\mathrm{n}$ & $\mathrm{n}$ & $\mathrm{n}$ & \\
\hline W4 & 224536 & 9.1 & 8.3 & 0.7 & 2 & 21 & $\mathrm{c}$ & F5V & $\ldots$ & $\mathrm{m}$ & $\mathrm{n}$ & $\mathrm{n}$ & \\
\hline W8 & & 10.1 & 4.9 & 0.6 & 3 & 21 & $\mathrm{c}$ & F6III & $\ldots$ & $\mathrm{m}$ & $\mathrm{n}$ & $\mathrm{n}$ & \\
\hline W9 & 224630 & 5.6 & -3.5 & 0.4 & 6 & 100 & $\mathrm{c}$ & K1II-III & $\ldots$ & $\mathrm{n}$ & $\mathrm{n}$ & $\mathrm{n}$ & \\
\hline W23 & 224820 & 8.4 & 4.8 & 2.2 & 6 & 27 & $\mathrm{c}$ & A1V & 180 & $\mathrm{~m}$ & $\mathrm{~m}$ & $\mathrm{~m}$ & \\
\hline W26 & 224862 & 7.1 & 17.6 & 0.4 & 4 & 65 & $\mathrm{c}$ & G6III & $\ldots$ & $\mathrm{n}$ & $\mathrm{n}$ & $\mathrm{n}$ & \\
\hline W28 & & 10.5 & 6.7 & 0.6 & 3 & 26 & $\mathrm{c}$ & F2-6V & $\ldots$ & $\mathrm{m}$ & $\mathrm{m}$ & $\mathrm{m}$ & \\
\hline W32 & & 11.1 & 10.7 & 0.7 & 4 & 24 & $\mathrm{c}$ & G8III & $\ldots$ & $\mathrm{n}$ & $\ldots$ & $\mathrm{n}$ & \\
\hline W35 & 224948 & 10.0 & 7.3 & 0.7 & 3 & 73 & $\mathrm{c}$ & F0V & 42 & $\mathrm{~m}$ & $\mathrm{~m}$ & $\mathrm{~m}$ & \\
\hline W36 & 224964 & 9.0 & 7.3 & 0.5 & 3 & 23 & $\mathrm{c}$ & A3V & 25 & $\mathrm{~m}$ & $\mathrm{~m}$ & $\mathrm{~m}$ & \\
\hline W37 & 224976 & 9.9 & 2.2 & 0.5 & 23 & 0 & $\mathrm{v}$ & F5V & 50 & $\mathrm{~m} ?$ & $\mathrm{~m}$ & $\mathrm{~m}$ & 1 \\
\hline W42 & 224990 & 5.0 & 5.5 & 0.7 & 95 & 0 & $\mathrm{v}$ & B4-5 & 28 & $\mathrm{~m}$ & $\mathrm{n}$ & $\mathrm{n}$ & 2 \\
\hline W47 & & 10.2 & 8.7 & 0.9 & 23 & 0 & $\mathrm{v}$ & $\mathrm{F} 2 \mathrm{~V}$ & 34 & $\mathrm{~m}$ & $\mathrm{~m}$ & $\mathrm{~m}$ & 2 \\
\hline W51 & 225047 & 8.4 & 6.6 & 2.0 & 5 & 87 & $\mathrm{c}$ & A0V & 120 & $\mathrm{~m}$ & $\mathrm{~m}$ & $\mathrm{~m}$ & \\
\hline W54 & & 10.4 & -4.7 & 0.8 & 4 & 38 & $\mathrm{c}$ & F1-5IV & 43 & $\mathrm{n}$ & $\mathrm{p}$ & $\mathrm{n}$ & 3,4 \\
\hline W55 & & 10.7 & 17.3 & 0.9 & 2 & 43 & $\mathrm{c}$ & G0 & $\ldots$ & $\mathrm{n}$ & $\mathrm{n}$ & $\mathrm{n}$ & 5 \\
\hline W56 & & 11.3 & 6.1 & 0.6 & 3 & 81 & $\mathrm{c}$ & F8 & $\ldots$ & $\mathrm{m}$ & $\mathrm{m}$ & $\mathrm{m}$ & 4 \\
\hline W57 & & 10.4 & 7.5 & 6.5 & 17 & 0 & $\mathrm{v}$ & F3V & 26 & $\mathrm{~m} ?$ & $\mathrm{~m}$ & $\mathrm{~m}$ & 2 \\
\hline W58 & & 10.6 & 6.7 & 0.6 & 5 & 89 & $\mathrm{c}$ & F8V & 56 & $\mathrm{~m}$ & $\mathrm{~m}$ & $\mathrm{~m}$ & \\
\hline W59 & 225099 & 9.4 & 8.0 & 0.5 & 2 & 33 & $\mathrm{c}$ & F5IV-V & $\ldots$ & $\mathrm{m}$ & $\mathrm{n}$ & $\mathrm{n}$ & \\
\hline W60 & & 10.6 & 6.7 & 0.5 & 5 & 14 & $\mathrm{c}$ & F4-6V & $\ldots$ & $\mathrm{m}$ & $\mathrm{m}$ & $\mathrm{m}$ & \\
\hline W61 & 225111 & 10.2 & 13.4 & 8.3 & 27 & 0 & $\mathrm{v}$ & F0V & 28 & $\mathrm{~m}$ & $\mathrm{~m}$ & $\mathrm{~m}$ & 2 \\
\hline W62 & & 10.4 & 18.9 & 0.9 & 2 & 74 & $\mathrm{c}$ & G6IV & $\ldots$ & $\mathrm{n}$ & $\mathrm{n}$ & $\mathrm{n}$ & \\
\hline W63 & & 10.6 & 4.6 & 0.4 & 5 & 36 & $\mathrm{c}$ & F4-6V & $\ldots$ & $\mathrm{m}$ & $\mathrm{m}$ & $\mathrm{m}$ & \\
\hline W65 & 225119 & 8.2 & 31.0 & 1.9 & 9 & 0 & $\mathrm{v}$ & B9: & 45 & $\mathrm{n}$ & $\mathrm{p}$ & $\mathrm{n}$ & 6 \\
\hline W69 & 225155 & 7.7 & -19.9 & 0.5 & 2 & 83 & $\mathrm{c}$ & G6III & $\ldots$ & $\mathrm{n}$ & $\mathrm{n}$ & $\mathrm{n}$ & \\
\hline W71 & 225187 & 7.1 & 1.9 & 2.8 & 13 & 0 & $\mathrm{v}$ & B7 & 150 & $?$ & $\mathrm{~m}$ & $\mathrm{~m} ?$ & \\
\hline W74 & & 10.5 & 6.6 & 0.5 & 10 & 53 & $\mathrm{c}$ & F6V & 53 & $\mathrm{~m}$ & $\mathrm{~m}$ & $\mathrm{~m}$ & \\
\hline W75 & 225200 & 6.4 & 14.9 & 5.0 & 12 & 0 & $\mathrm{v} ?$ & A2shell & 300: & m: & $\mathrm{p}$ & $\mathrm{m} ?$ & 7 \\
\hline W76 & 225206 & 7.8 & 6.0 & 1.8 & 40 & 0 & $\mathrm{v}$ & $\mathrm{B} 8 \mathrm{~V}$ & 146 & $\mathrm{~m}$ & $\mathrm{~m}$ & $\mathrm{~m}$ & \\
\hline W80 & & 11.0 & 7.7 & 0.6 & 5 & 26 & $\mathrm{c}$ & F6-8 & $\ldots$ & $\mathrm{m}$ & $\mathrm{m}$ & $\mathrm{m}$ & \\
\hline W86 & 225264 & 8.3 & 5.0 & 3.4 & 16 & 0 & $\mathrm{v}$ & A0-A1V & 33 & $\mathrm{~m}$ & $\mathrm{~m}$ & $\mathrm{~m}$ & 2 \\
\hline W88 & 225282 & 8.3 & 5.4 & 1.4 & 8 & 86 & $\mathrm{c}$ & $\mathrm{A} 0-1$ & 137 & $\mathrm{~m}$ & $\mathrm{~m}$ & $\mathrm{~m}$ & 3 \\
\hline W96 & 50 & 9.7 & 4.5 & 1.9 & 32 & 0 & $\mathrm{v}$ & F0V & 40 & $\mathrm{~m}$ & $\mathrm{~m}$ & $\mathrm{~m}$ & \\
\hline W104 & 91 & 9.9 & 6.8 & 0.5 & 2 & 78 & $\mathrm{c}$ & F0V & $\ldots$ & $\mathrm{m}$ & $\mathrm{m}$ & $\mathrm{m}$ & 2 \\
\hline W106 & 104 & 8.0 & -0.3 & 0.6 & 2 & 80 & $\mathrm{c}$ & G8-K0III & $\ldots$ & $\mathrm{n}$ & $\mathrm{n}$ & $\mathrm{n}$ & \\
\hline W109 & 117 & 9.2 & 15.7 & 0.5 & 4 & 99 & $\mathrm{c}$ & F4-8 & $\ldots$ & $\mathrm{n}$ & $\mathrm{n}$ & $\mathrm{n}$ & \\
\hline W111 & 141 & 7.9 & 11.4 & 4.4 & 8 & 100 & $\mathrm{c}$ & B9V & 204 & $\mathrm{~m}$ & $\mathrm{~m}$ & $\mathrm{~m}$ & \\
\hline W121 & 212 & 10.2 & 7.7 & 2.1 & 5 & 1 & $?$ & F3V & 94 & $\mathrm{~m}$ & $\mathrm{~m}$ & $\mathrm{~m}$ & \\
\hline W124 & 235 & 8.7 & 4.9 & 4.3 & 11 & 100 & $\mathrm{c}$ & A1V & 218 & $\mathrm{~m}$ & $\mathrm{~m}$ & $\mathrm{~m}$ & 3 \\
\hline W126 & 343 & 9.8 & 7.5 & 5.7 & 3 & 2 & $?$ & A6 & 155 & $\mathrm{~m}$ & $\mathrm{~m}$ & $\mathrm{~m}$ & \\
\hline W128 & 394 & 9.6 & 3.9 & 1.1 & 2 & 94 & $\mathrm{c}$ & F8V & 34 & $\mathrm{~m}$ & $\mathrm{n}$ & $\mathrm{n}$ & \\
\hline E54 & & 10.1 & 5.4 & 0.5 & 3 & 83 & $\mathrm{c}$ & F0V & 28 & $\mathrm{~m}$ & $\mathrm{~m}$ & $\mathrm{~m}$ & 3 \\
\hline E57 & 225077 & 8.9 & 7.5 & 1.2 & 28 & 0 & $\mathrm{v}$ & $\mathrm{A} 3 \mathrm{~V}$ & $\ldots$ & $\mathrm{m}$ & $\mathrm{m}$ & $\mathrm{m}$ & 2 \\
\hline E51 & & 10.3 & 5.2 & 1.1 & 3 & 86 & $\mathrm{c}$ & F5V & 86 & $\mathrm{~m}$ & $\mathrm{~m}$ & $\mathrm{~m}$ & \\
\hline
\end{tabular}

Notes: 1 the location in the color-magnitude diagram suggests duplicity (see Fig. 2); 2 binary star with calculated orbit. For kinematic membership $V_{\gamma}$ was used; $3 \mathrm{RV}$ variability inferred from the RV difference with previous studies (see text); 4 proper motion membership taken from Mermilliod et al. (2008); 5 RV variable in Mermilliod et al. (2008); 6 suspected SB2 (see text); 7 Shell Star. RV of narrow spectral lines is constant at $6.6 \pm 0.7 \mathrm{~km} \mathrm{~s}^{-1}$.

W42: $\zeta \mathrm{Scl}$ is the brightest star in the region. Its RV is not far from the cluster mean, and in the HR diagram it is near the ZAMS, above the turnoff point, in a position typical of blue straggler stars. However, based on Hipparcos proper motions, Baumgardt (2000) assigns a membership probability of $0 \%$ to this star; the proper motion difference with the cluster mean is about 10 mas/yr. The residuals of the fitted orbit are larger than the measurement errors. Repeated observations during the same run indicate the presence of low-amplitude short-period variations.
W47: it is a low-amplitude long-period variable, and the orbital parameters included in Table 3 are not definitive, and even the period should be confirmed with additional observations. The fitted orbit is indistinguishable from circular. However, we have not fixed the eccentricity at zero value since, which such a long period, there is no reason to assume that it is circularized. The only published RV measurement for this star $\left(0.3 \pm 0.4 \mathrm{~km} \mathrm{~s}^{-1}\right.$, Edvardsson et al. 1995) is consistent with our orbit. Including this point in the fitting would suggest a slightly higher eccentricity $(e=0.14)$ and an argument of periastron $\omega=1.7 \mathrm{rad}$. 
Table 3. Orbital parameters of spectroscopic binaries.

\begin{tabular}{|c|c|c|c|c|c|c|c|c|c|}
\hline Star & $\begin{array}{r}T_{\text {per }} \\
-2450000\end{array}$ & $\begin{array}{r}T_{\text {conj }} \\
-2450000\end{array}$ & $\begin{array}{r}V_{\gamma} \\
\mathrm{km} \mathrm{s}^{-1}\end{array}$ & $\begin{array}{r}K \\
\mathrm{~km} \mathrm{~s}^{-1}\end{array}$ & $\begin{array}{r}\omega \\
\mathrm{rad}\end{array}$ & $e$ & $\begin{array}{l}P \\
\mathrm{~d}\end{array}$ & $\begin{array}{c}\sigma \\
\mathrm{km} \mathrm{s}^{-1}\end{array}$ & $\bar{N}$ \\
\hline \multirow[t]{2}{*}{ W42 } & 3381 & 3498 & 5.29 & 12.4 & 0.76 & 0.316 & 1740 & 1.7 & 95 \\
\hline & \pm 37 & \pm 17 & \pm 0.34 & \pm 0.6 & \pm 0.12 & \pm 0.043 & \pm 22 & & \\
\hline \multirow[t]{2}{*}{ W47 } & 4839 & 5040 & 6.81 & 6.9 & 0.4 & 0.12 & 1338 & 1.0 & 23 \\
\hline & \pm 344 & \pm 52 & \pm 0.41 & \pm 1.3 & \pm 1.6 & \pm 0.19 & \pm 71 & & \\
\hline \multirow[t]{2}{*}{ W57 } & 3602.33 & 3601.440 & 3.96 & 44.53 & 3.94 & 0.029 & 2.403362 & 0.60 & 17 \\
\hline & \pm 0.09 & \pm 0.005 & \pm 0.20 & \pm 0.29 & \pm 0.22 & \pm 0.006 & \pm 0.000019 & & \\
\hline \multirow[t]{2}{*}{ W61 } & $\ldots$ & 3501.1397 & 7.47 & 52.84 & $\ldots$ & 0.000 & 1.923291 & 1.4 & 27 \\
\hline & $\ldots$ & \pm 0.0024 & \pm 0.27 & \pm 0.35 & $\ldots$ & 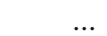 & \pm 0.000010 & & \\
\hline \multirow[t]{2}{*}{ W86 } & 3689.782 & 3689.667 & 5.23 & 19.15 & 8.10 & 0.267 & 5.400945 & 0.34 & 16 \\
\hline & \pm 0.029 & \pm 0.012 & \pm 0.10 & \pm 0.15 & \pm 0.03 & \pm 0.008 & \pm 0.000040 & & \\
\hline \multirow[t]{2}{*}{ W96 } & 3503.05 & 3506.59 & 6.66 & 14.96 & 6.88 & 0.387 & 51.416 & 0.7 & 32 \\
\hline & \pm 0.42 & \pm 0.20 & \pm 0.17 & \pm 0.29 & \pm 0.05 & \pm 0.015 & \pm 0.007 & & \\
\hline \multirow[t]{2}{*}{ E57 } & 3786.8 & 3735.9 & 5.0 & 11.9 & 4.22 & 0.623 & 191.41 & 0.7 & 28 \\
\hline & \pm 5.2 & \pm 5.2 & \pm 0.5 & \pm 1.1 & \pm 0.02 & \pm 0.023 & \pm 0.40 & & \\
\hline \multirow[t]{2}{*}{ HD 224113A } & 3782.811 & 3782.053 & 2.5 & 84.8 & 3.6 & 0.059 & 2.44508387 & 2.7 & 11 \\
\hline & \pm 0.081 & \pm 0.021 & \pm 1.9 & \pm 3.5 & \pm 0.4 & \pm 0.029 & $\ldots$ & & \\
\hline HD 224113B & & & & 191.5 & & & & 9.2 & \\
\hline
\end{tabular}

W61: the spectroscopic orbit is indistinguishable from circular, with an eccentricity $e=0.0066 \pm 0.0074$. In the final fitting, corresponding to the orbital parameters tabulated in Table 3 , the eccentricity was fixed at zero. It has a visual companion (W62, $V=10.4)$ at 55.6 arcsec.

W65: the RV of this star is clearly variable, but there are not enough observations to determine the period of these variations. The peak profile in the cross-correlation function indicates that it is possibly an SB2, although one not easy to resolve. The FWHM of the spectral lines are about $66 \mathrm{~km} \mathrm{~s}^{-1}$ and $51 \mathrm{~km} \mathrm{~s}^{-1}$ for the primary and secondary line sets, respectively, while the maximum RV difference between the components is approximately $50 \mathrm{~km} \mathrm{~s}^{-1}$ in our spectra. The measurement of $\mathrm{RVs}$, assuming it is an SB2, gives a mass ratio $q=0.57$ and a center-of-mass velocity $V_{\gamma}=29 \mathrm{~km} \mathrm{~s}^{-1}$. It is a non-member according to the mean RVs, which is supported by its location in the color-magnitude diagram, to the left of the main sequence $(V=8.19, B-V=-0.12)$.

W71: it is a long-period variable. The observations taken during three different runs show an RV increasing at the rate of about $3 \mathrm{~km} \mathrm{~s}^{-1} \mathrm{yr}^{-1}$. The average $\mathrm{RV}$ of the spectra taken during the years 2002, 2003, and 2008 are $-6.5 \pm 2.1 \mathrm{~km} \mathrm{~s}^{-1},-1.1 \pm$ $2.7 \mathrm{~km} \mathrm{~s}^{-1}$, and $+13.1 \pm 2.2 \mathrm{~km} \mathrm{~s}^{-1}$, respectively. The variable character of this star is also supported by the three old measurements by Buscombe (1963), which differ from each other by several tens of $\mathrm{km} \mathrm{s}^{-1}$. For this reason, it is not possible to assign kinematic membership to this star. Its proper motion and its location in the color-magnitude diagram are compatible with cluster membership, so we consider that it could be a binary star member of the cluster. This star is a low-amplitude photometric variable with $\Delta V=0.03$ mag (Schneider 1987).

W75: this is a shell star, presenting a broad line spectrum with sharp lines of Fe II (e.g. $\lambda \lambda 4233,4549,4584,4923,5018)$. This would explain its location to the right of the cluster main sequence. To determine the stellar RV, we measured only the Mg II spectral line $\lambda 4481$. This star is a possible RV variable, although the uncertainty of these measurements is rather large.
The RVs of Buscombe (1963) also indicate variability. On the contrary, the RV of the shell lines is constant with a mean value very close to the cluster RV.

W76: it presents a broad line spectrum. The peak profile in the cross-correlation function is variable and sometimes asymmetrical. The rms of the measured $\mathrm{RVs}$ is $10.5 \mathrm{~km} \mathrm{~s}^{-1}$. The best orbital fit, corresponding to a period of 33.128 days and a semi-amplitude of $14 \mathrm{~km} \mathrm{~s}^{-1}$, gives an rms of the residuals of $5.5 \mathrm{~km} \mathrm{~s}^{-1}$. Other periods are also compatible with the observational data, for example 1.0283 days $\left(\mathrm{rms}=6.7 \mathrm{~km} \mathrm{~s}^{-1}\right)$, 36.27 days $\left(\mathrm{rms}=7.7 \mathrm{~km} \mathrm{~s}^{-1}\right)$, and 1029. days $(\mathrm{rms}=$ $6.3 \mathrm{~km} \mathrm{~s}^{-1}$ ). The period is not unambiguously determined, even when 40 measurements have been obtained, because of the low amplitude of RV variations, which is about one tenth of the spectral lines width. Such small RV variations and the profile variations mentioned above could be caused by a rotating spotted star. In that case the period is expected to be about one day or shorter, according to the observed rotational velocity. If the long period is correct, then the profile variations could be interpreted as the result of blending lines of two fast-rotating stellar companions.

W86: this is a quite short period binary with an eccentric orbit; therefore, these properties make it an important target for tests of circularization theories.

HD 224113 and HD 224112: these objects are two bright stars ( $V=6.1$ and $V=6.8$, respectively) located at $2.7 \mathrm{deg}$ from the cluster center. We observed these stars as part of the sample of stars in a region around the cluster. Even though they have not been considered as cluster members, it is worth discussing the possibility of their membership since they are early-type stars similar to $\zeta \mathrm{Scl}$.

These two stars form a visual pair with a separation of 134 arcsec. They share the same proper motion of the cluster Blanco 1 and the distance derived from the Hipparcos parallax is for both stars $d=310_{-65}^{+110} \mathrm{pc}$, in agreement with the cluster distance, within the uncertainties.

HD 224113 is an eclipsing binary (AL Scl) with a period of 2.44 days. It was reported as an SB2 with a low-luminosity 

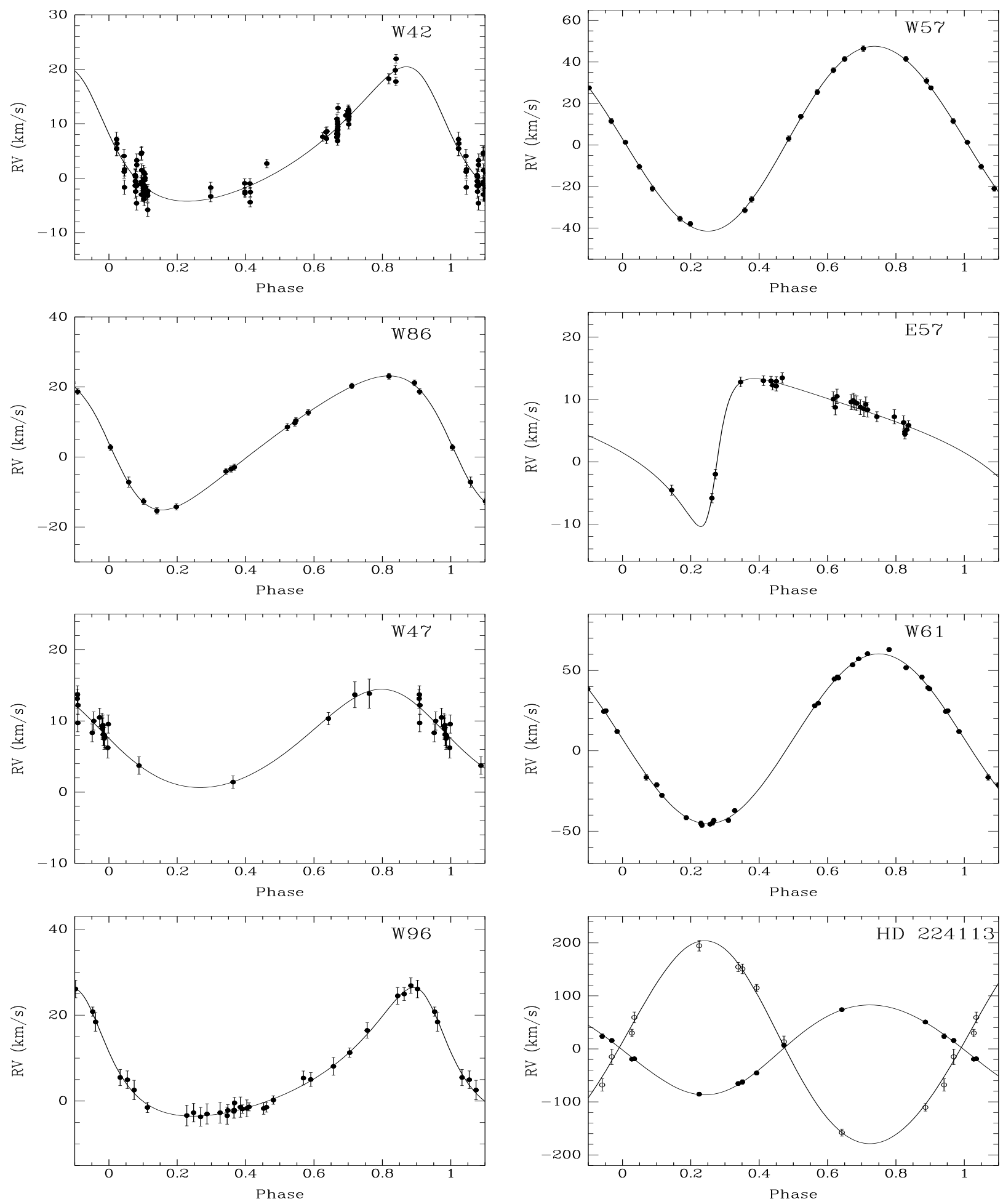

Fig. 1. Radial velocity curves of 8 spectroscopic binaries. Labels in the upper right corner of each panel are star identification numbers. Zero phase corresponds to the primary conjunction.

ratio by Haefner et al. (1987), who identified the secondary component only in the Ca II line 23933 . These authors noted that the center-of-mass velocity changes with time (between -2.6 and $+10.4 \mathrm{~km} \mathrm{~s}^{-1}$ ), so it is not possible to evaluate its kinematic membership. In our observations, the center-of-mass RV is $2.5 \pm$ $1.9 \mathrm{~km} \mathrm{~s}^{-1}$. On the other hand, HD 224112 did not show variations in our observations, and its mean $\mathrm{RV}$ is $+3.5 \pm 1.0 \mathrm{~km} \mathrm{~s}^{-1}$.
Finally we note that the absolute parameters derived from RV and light curves by Haefner et al. (1987) for HD 224113 are compatible with the cluster age. In summary, this is a multiple star - a quadruple if we consider the presence of a third body around the eclipsing component - that could be related to Blanco 1. The main reason to consider this star as a non-member is the large angular distance to the cluster, which would be longer 
than the cluster tidal radius. However, we speculate that this may be one of the most massive stars of the cluster that has recently escaped from the cluster potential.

\section{Cluster kinematics and membership}

Most of the stars studied show a mean RV between 4 and $9 \mathrm{~km} \mathrm{~s}^{-1}$ (see Tables 1 and 2), clearly identifying the cluster velocity. To derive the mean cluster velocity, we calculated the average of the RV of the member stars.

In the case of spectroscopic binaries we use the center-ofmass velocity, and RV variables without orbits were not considered. To take the uncertainty of the mean RV of each star into account, we assigned weights defined as $\left[\operatorname{err}^{2}+\left(1.0 \mathrm{~km} \mathrm{~s}^{-1}\right)^{2}\right]^{-1}$. This strategy avoids overweighting the stars whose mean RV are more precisely defined. The reason for such a definition is that the RV of one star of the cluster can be considered as a measurement of the mean cluster velocity with an uncertainty not lower than the internal velocity dispersion of the cluster.

We defined as member stars those for which the quadratic sum of the error and the velocity dispersion of the cluster is greater than the absolute difference between the mean RV of the star and that of the cluster. We iterate between the determination of the cluster parameters (mean velocity $\langle\mathrm{RV}\rangle_{\mathrm{cl}}$ and dispersion $\sigma_{\mathrm{cl}}$ ), and the identification of cluster members. Finally, the cluster velocity was calculated with 21 stars with high membership probability and small RV error $\left(\leq 2 \mathrm{~km} \mathrm{~s}^{-1}\right)$ :

$\langle\mathrm{RV}\rangle_{\mathrm{cl}}=6.2 \pm 0.3 \mathrm{~km} \mathrm{~s}^{-1}$

$\sigma_{\mathrm{cl}}=1.3 \mathrm{~km} \mathrm{~s}^{-1}$.

These membership determinations were complemented with available proper motion data. We used the catalogs Hipparcos (ESA 1997), UCAC2 (Zacharias et al. 2004), SMP3 (Girard et al. 2004), and PPM (Bastian \& Roeser 1993) to calculate the mean proper motion $\mu_{\alpha}^{\mathrm{cl}}$ and $\mu_{\delta}^{\mathrm{cl}}$ of the cluster. Then, we defined normalized star-cluster differences as

$\rho=\left[\frac{\left(\mu_{\alpha}-\mu_{\alpha}^{\mathrm{cl}}\right)^{2}}{\left(\Delta \mu_{\alpha}^{2}+\Delta \mu_{\alpha}^{\mathrm{cl}}\right)^{2}}+\frac{\left(\mu_{\delta}-\mu_{\delta}^{\mathrm{cl}}\right)^{2}}{\left(\Delta \mu_{\delta}^{2}+\Delta \mu_{\delta}^{\mathrm{cl}}\right)^{2}}\right]^{1 / 2}$

where $\Delta \mu$ and $\Delta \mu^{\mathrm{cl}}$ represent the uncertainty in the proper motion of the star (error assigned in the catalog) and the cluster (calculated from the point dispersion). Proper motion memberships were defined as members $\rho<2$, probable members $2<\rho<3$, and non-members $\rho>3$.

Finally, we combined the spectroscopic and astrometric membership to assign a member classification (last column in Table 2). Figure 2 shows the color-magnitude diagram for all stars in our sample, distinguishing membership status and binarity. Stars classified as member by Mermilliod et al. (2008) have also been included in these diagrams. We plotted an isochrone for solar composition corresponding to $\log ($ age $)=8.2$, and cluster parameters $V-M_{v}=7.1$, and $E(B-V)=0.01$. Stellar models were taken from the Girardi et al. (2000) grids. The adopted distance modulus was chosen to fit the main sequence of the cluster members in Fig. 2 and is consistent with previous works (6.99, Westerlund et al. 1988; 7.18, Kharchenko et al. 2005). The age of the cluster is not well-defined because of the lack of stars close to the turnoff point (i.e. close to the TAMS). All the early photometric studies (Eggen 1979; Perry et al. 1978; Abraham de Epstein \& Epstein 1985) found the age of Blanco 1 to be very similar to the Pleiades, which is now accepted to have an age of about

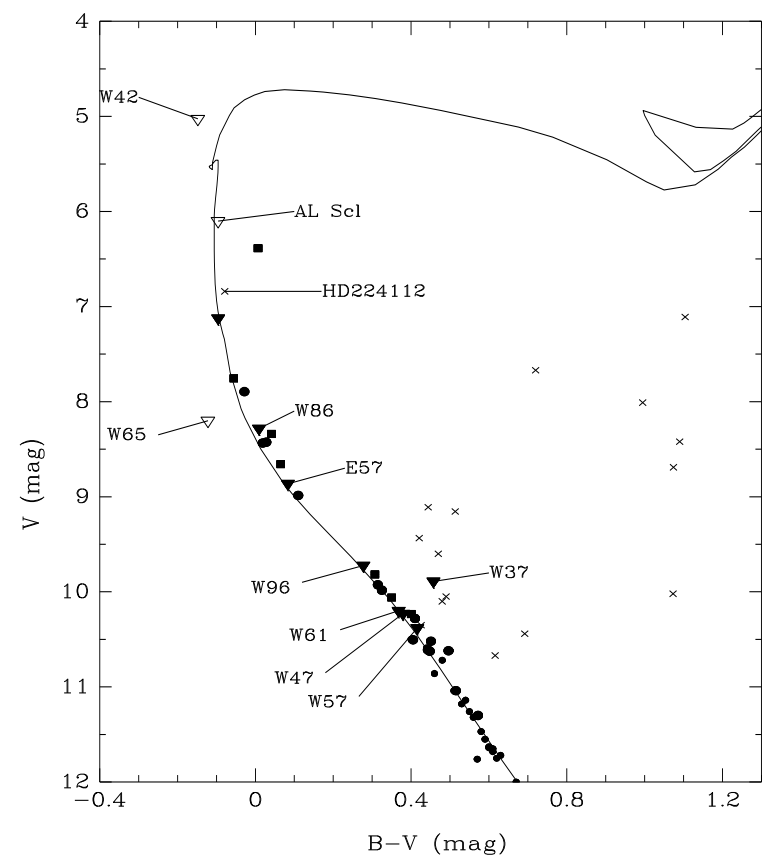

Fig. 2. Color-magnitude diagram. Filled triangles: member binaries; open triangles: field binaries; filled squares: probable binary members; filled circles: constant-velocity kinematics members; crosses: single non-members; small filled circles: members from Mermilliod et al. (2008).

$\log ($ age $)=8.1$. The catalog of Kharchenko et al. (2005) adopts $\log ($ age $)=8.32$ for Blanco 1 . We have adopted here the value $\log ($ age $)=8.2$.

With the only exception of W37, all binary stars are very close to the cluster main sequence, which would indicate that their secondary components do not contribute significantly to the total light of the systems. This agrees with the secondary spectral lines not being visible in our spectra.

The position of the eclipsing binary AL Scl and its visual companion HD 224112 are in agreement with the cluster sequence. This is not affected by the light of the secondary companion of AL Scl, which contributes a small fraction of the total light. In fact, since $q=0.54$, the luminosity ratio is expected to be $L_{2} / L_{1} \approx 0.1$.

\section{Discussion}

We have obtained spectroscopic observations of 69 stars in the field of the open cluster Blanco 1 and its surroundings, covering a wide range of spectral types in the main sequence from B4 to F7. Among cluster members we found 14 RV-constant stars, 9 SB1s, 3 suspected variable stars, and 3 stars whose variability was deduced from the comparison of our RVs with those obtained in previous publications. Therefore, in our sample of 29 members of Blanco 1, the binary frequency is $41-52 \%$.

The mean cluster RV was calculated from RV-constant members and binaries with calculated orbit: $\langle\mathrm{RV}\rangle_{\mathrm{cl}}=6.2 \pm$ $0.3 \mathrm{~km} \mathrm{~s}^{-1}$. Our mean radial velocity is slightly higher than published by Jeffries \& James (1999) $\left(5.0 \pm 0.2 \mathrm{~km} \mathrm{~s}^{-1}\right)$ and Mermilliod et al. (2008) $\left(5.53 \pm 0.11 \mathrm{~km} \mathrm{~s}^{-1}\right)$. Our star sample has 13 stars in common with the work of Mermilliod et al., 8 of which are RV constant stars. Using the average velocity for these 8 stars, we estimated that there is effectively a systematic difference of about $1.5 \mathrm{~km} \mathrm{~s}^{-1}$. This difference, larger than the 
formal uncertainties, could in part come from low-amplitude variables not detected in the sample or systematic instrumental errors, but also from the definition of the zero point and the gravitational redshift. As described above, our velocity zero point was established using synthetic spectra. The measurement of some standard stars from the list of Udry et al. (1999) observed in some of our runs shows the same trend: the RV difference with published radial velocity is typically $0.9 \mathrm{~km} \mathrm{~s}^{-1}$. We consider that part of that difference is related to the gravitational redshift that is not included in the zero point definition of the standard system, which is based on the solar RV (observation of minor planets). The RV of the Sun, measured in an absolute system, is not zero but about $0.64 \mathrm{~km} \mathrm{~s}^{-1}$, the solar gravitational redshift. The gravitational redshift varies along the main sequence, being greater than $1 \mathrm{~km} \mathrm{~s}^{-1}$ for B-type stars.

We have determined orbits for seven new spectroscopic binaries with periods in the range 1.9-1572 days. We note that all our cluster binary stars have single-lined spectra, which indicates that the luminosity-ratio is around 0.1 or lower. Assuming a mass-luminosity relation with a power law with index 4 , this would imply that there are no binary stars with mass ratio $q \geq$ 0.56 among cluster stars in our sample.

The binary population of Blanco 1 constitutes an important piece of information for the confrontation of models of binary circularization. Star W86, with a period of 5.40 days, has an eccentric orbit $(e=0.27)$ and the two binaries with periods below 2.4 days (W57 and W61) have nearly circular orbits. Particularly interesting is the binary W86, since its circularization time scale is close to the cluster age. A detailed calculation of the circularization times requires an estimate of the stellar parameters of the binary components. As a rough estimate, however, we calculated the circularization time-scale using the primary mass and radius derived from the color-magnitude diagram and assuming the mass ratio to be $q \approx 0.1-0.4$. Using the algorithms of Claret et al. (1995) for the tidal theory by Tassoul $(1987,1988)$ and adopting an age of $10^{8.2} \mathrm{yr}$ for the cluster, we calculate that the original eccentricity of the stars W86, W57, and W61 has diminished approximately by a factor $e / e_{o}=0.8,0.19$, and 0.01 , respectively. These values are consistent with the present eccentricity of these systems.

Finally, we noted that the eclipsing binary AL Scl, along with its visual companion, could constitute a multiple system connected to the cluster since, despite its large distance to the cluster center $(2.7 \mathrm{deg})$, photometric and kinematic criteria would give it a high membership probability.
Acknowledgements. This paper was partially supported by a grant from CONICET PIP 11220080101113. We are grateful to the technical staff of the CASLEO, particularly to P. Ostrov, A. De Franceschi, and R. Jackowzyk, who carried out part of the observations analyzed here. Some spectra were kindly taken by R. Gamen and M. E. Veramendi.

\section{References}

Abraham de Epstein, A. I., \& Epstein, I. 1985, AJ, 90, 1211

Bastian, U., \& Roeser, S. 1993, Positions and Proper Motions - South, Astronomisches Rechen-Institut, Heidelberg

Blanco, V. M. 1949, PASP, 61, 183

Bond, H. E., \& Perry, C. L. 1971, PASP, 83, 643

Baumgardt, H., Dettbarn, C., \& Wielen, R. 2000, A\&AS, 146, 251

Buscombe, W. 1963, MNRAS, 126, 29

Cargile, P. A., James, D. J., \& Platais, I. 2009, AJ, 137, 3230

Claret, A., Giménez, A., \& Cunha, N. C. S. 1995, A\&A, 299, 724

Edvardsson, B., Pettersson, B., Kharrazi, M., \& Westerlund, B. 1995, A\&A, 293, 75

Epstein, I. 1968, AJ, 73, 556

ESA 1997, The Hipparcos and Tycho Catalogues, ESA SP-1200 (Noordwijk: ESA)

Ford, A., Jeffries, R. D., \& Smalley, B. 2005, MNRAS, 364, 282

Girardi, L. O., Bressan, A., Bertelli, G., \& Chiosi, C. 2000, A\&A, 141, 371

González, J. F., \& Levato, H. 2006a, RMxAC, 26, 171

González, J. F., \& Levato, H. 2006b, A\&A, 448, 283

Jeffries, R. D., \& James, D. J. 1999, ApJ, 511, 218

Haefner, R., Skillen, I., \& de Groot, M. 1987, A\&A, 179, 141

Levato, H., González, J. F., Malaroda, S., \& Grosso, M. 2004, RMxAC, 21, 141

Mermilliod, J. C., Platais, I., James, D. J., Grenon, M., \& Cargile, P. A. 2008, A\&A, 485, 95

Micela, G., Sciortino, S., Favata, F., Pallavicini, R., \& Pye, J. 1997, Mem. Soc. Ast. It., 68

Micela, G., Sciortino, S., Favata, F., Pallavicini, R., \& Pye, J. 1999, A\&A, 344, 96

Moraux, E., Bouvier, J., Stauffer, J. R., Barrado y Navascués, D., \& Cuillandre, J.-C. 2007, A\&A, 471, 513

Munari, U., Sordo, R., Castelli, F., \& Zwitter, T. 2005, A\&A, 442, 1127

Panagi, P. M., O'dell, M. A., Collier Cameron, A., \& Robinson, R. D. 1994, A\&A, 292, 449

Pillitteri, I., Micela, G., Sciortino, S., \& Favata, F. 2003, A\&A, 399, 929

Pillitteri, I., Micela, G., Sciortino, S., Damiani, F., \& Harnden, F. R., Jr. 2004, A\&A, 421, 186

Pillitteri, I., Micela, G., Reale, F., \& Sciortino, S. 2005, A\&A, 430, 164

Platais, I., Girard, T. M., Kozhurina-Platais, V., et al. 2004, AJ, 127, 3060

Randich, S. 1997, Mem. Soc. Ast. It., 68,

Schneider, H. 1987, Hv. Obs. Bull., 11, 29

Tassoul, J. L. 1987, ApJ, 322, 856

Tassoul, J. L. 1988, ApJ, 324, L71

Udry, S., Mayor, M., \& Queloz, D. 1999, ASPC, 185, 367

Westerlund, B. E., Lundgren, K., Petterson, B., Garnier, R., \& Breysacher, J. 1988, A\&AS, 76, 101

Zacharias, N., Urban, S. E., Zacharias, M. I., et al. 2004, AJ, 127, 3043 